\title{
Trends in total fat and fatty acid intakes and chronic health conditions in Korean adults over 2007-2015
}

\author{
SuJin Song ${ }^{1}$, Jae Eun Shim ${ }^{2, *}$ and Won $O$ Song $^{3}$ \\ 'Department of Food and Nutrition, Hannam University, Daejeon, South Korea: ${ }^{2}$ Department of Food and Nutrition, \\ Daejeon University, 62 Daehak-ro, Dong-gu, Daejeon 34520, South Korea: ${ }^{3}$ Department of Food Science and \\ Human Nutrition, Michigan State University, East Lansing, MI, USA
}

Submitted 23 June 2018: Final revision received 17 October 2018: Accepted 19 November 2018: First published online 15 February 2019

\begin{abstract}
Objective: To investigate trends in total fat and fatty acid intakes and chronic health conditions among Korean adults over nine years between 2007 and 2015.

Design: Cross-sectional, observational study using a stratified, multistage probability sampling design at a national level. Intakes of total fat and fatty acids were estimated from $24 \mathrm{~h}$ dietary recalls by sex and age groups. Trends of total fat and fatty acid intakes were determined by multiple linear regression after adjusting for covariates. Trends in age-standardized prevalence of obesity, hypercholesterolaemia and hypertriacylglycerolaemia were examined by sex.

Setting: Korea.

Participants: Population data of 47749 healthy adults (aged $\geq 19$ years) derived from the Korea National Health and Nutrition Examination Survey between 2007 and 2015.

Results: Over the survey period, daily intakes of energy and total, saturated, monounsaturated, polyunsaturated, $n-3$ and $n-6$ fats (grams and percentage of energy (\%E)) increased steadily. In all sex and age groups, significant increases were seen in SFA intake from $9.9 \mathrm{~g}(4.7 \% \mathrm{E})$ to $12.0 \mathrm{~g}(5.3 \% \mathrm{E})$ and in MUFA intake from $9.9 \mathrm{~g}(4.6 \% \mathrm{E})$ to $13.3 \mathrm{~g}(5 \cdot 8 \% \mathrm{E})$. The prevalence of hypercholesterolaemia increased from $10 \cdot 7$ to $17 \cdot 9 \%$ over the same period.

Conclusions: In Korean adults, total fat, SFA and other fatty acids have been increasing along with the prevalence of hypercholesterolaemia. This information can help set adequate macronutrient and fatty acid distribution ranges in developing population-specific preventive strategies against diet-related illness.
\end{abstract}

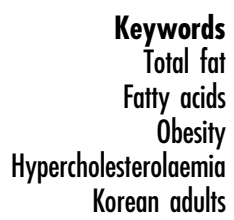

For the past few decades, non-communicable diseases (NCD) such as CHD, stroke, diabetes and cancers have been leading causes of mortality and disability worldwide. Elevated LDL-cholesterol, high blood pressure, being overweight or obese, and smoking have been established as modifiable risk factors for $\mathrm{CHD}^{(1)}$. Several classical reports supported that total fat and saturated fat intakes significantly increase the blood LDL-cholesterol level ${ }^{(2)}$. Therefore, dietary fat, particularly saturated fat, has been considered a potent dietary risk factor for CHD.

Recently systematic reviews of randomized controlled trials and prospective cohort studies have reported that saturated fat intake is not associated with $\mathrm{CVD}^{(3-5)}$. However, the randomized controlled trials and prospective cohort studies included in those systematic reviews did not account for the nutrients that replaced the fats or saturated fats ${ }^{(6-8)}$. Several other studies have pointed out that the reduction of dietary fats is often accomplished by replacing of saturated fats with carbohydrates, especially sugars, which results in obesity and other related diseases $^{(9)}$. A number of other studies have indicated that dietary saturated fat, in contrast to complex carbohydrates and cis-unsaturated fatty acids, raises LDLcholesterol and, in turn, the risk of $\mathrm{CHD}^{(10,11)}$. Thus, the impact of modifying one's diet with a focus on fat composition still needs to be examined.

The worldwide increase in diet-related NCD has been accompanied by the increased consumption of more energy-dense and sweeter foods ${ }^{(12)}$. The increased consumption of animal-source foods, main sources of saturated fat and cholesterol, has also played an important role in the changes of dietary patterns ${ }^{(13)}$. Efforts to improve dietary intakes in certain sociodemographic subgroups of Western countries have somewhat improved diet-related health problems, while the situation has persisted in other subgroups of such countries as well as in other parts of 
developing countries, where a double burden of inadequate nutrient intake and high consumption of energy and fat exists with diet-related $\mathrm{NCD}^{(14)}$.

The current disparity in eating patterns and health statuses among various race/ethnicity groups, regions and countries suggests the need for differentiated approaches that are specific to different diet and health contexts. In Korea, heart disease is the second-leading cause of mortality. Scientists and policy makers in Korea have debated the ideal macronutrient intake levels to prevent dietrelated illness, but estimations of population-level consumption of different types of fat have not been possible thus far due to lack of a comprehensive fatty acid food composition database. Recently, a fatty acid database was constructed for the preparation of dietary data from the Korea National Health and Nutrition Examination Survey (KNHANES $^{(15)}$, which enabled us to conduct the present study. In the present study, trends in total and types of fat intake, macronutrient contributions to energy and health conditions (i.e. obesity and dyslipidaemia) were investigated among Korean adults. The study used a valid and comprehensive database to document novel evidence for preventive strategies against diet-related illness such as CHD.

\section{Methods}

\section{Data and participants}

The present study was based on data from the fourth (2007-2009), fifth (2010-2012) and sixth (2013-2015) KNHANES. The KNHANES is a national survey designed to assess and monitor the health and nutritional statuses of the Korean population at the national level. It is a crosssectional and nationally representative survey that is conducted every year by the Korea Centers for Disease Control and Prevention. The survey uses a stratified, multistage probability sampling design and consists of three survey sections: health interview, health examination and nutrition survey. Detailed explanations of the KNHANES are available elsewhere ${ }^{(16)}$.

Among the eligible individuals who were aged 19 years or older and had dietary data ( $n$ 49649), we excluded individuals who were pregnant or breast-feeding ( $n$ 927) or reported extreme energy intakes, defined in the present study as <1st or $>99$ th percentile of daily energy intake by sex ( $n$ 973). A total of 47749 participants (20 024 men and 27725 women) was included in the final data analyses. The study was conducted according to the guidelines laid out in the Declaration of Helsinki and all procedures involving human subjects were approved by the Korea Centers for Disease Control and Prevention Institutional Review Board. Written informed consent was obtained from all subjects.

\section{Dietary assessment}

Dietary intake data were obtained by a single 24 h dietary recall. The dietary data were collected by trained dietitians at each participant's home, one week after the completion of the health interview survey and health examination survey. Energy and macronutrient intakes were calculated for each participant using the food composition table published by the Korea Rural Development Administration $^{(17,18)}$. Energy and macronutrient intakes were presented as absolute amounts in grams as well as proportions (i.e. percentage of total energy intake (\%E)). Fatty acid intakes in the sixth KNHANES were calculated using a new database which was developed in $2014^{(15)}$. This database contains the fatty acid contents of 5144 Korean foods items used in the KNHANES data. Analytical values of fatty acid contents in foods were compiled from domestic and foreign sources based on the evaluation of data quality. In cases of missing values, the fatty acid contents were calculated or imputed based on the analytical values of similar foods. The process of developing the database is described in detail elsewhere ${ }^{(15)}$. At the time of the fourth and fifth KNHANES surveys, fatty acid composition tables were not available. Thus, fatty acid intakes in the fourth and fifth KNHANES were calculated using the fatty acid contents per $100 \mathrm{~g}$ of foods which were replaced with the sixth KNHANES data. For foods which did not appear in the sixth KNHANES, the fatty acid contents were replaced with calculated or imputed values using the fatty acid contents of similar foods in the sixth KNHANES data or in the US Department of Agriculture fatty acid database ${ }^{(19)}$. Regarding mixed foods, the fatty acid contents of the main food source for total fat were applied.

\section{Chronic bealth conditions}

Information on chronic health conditions was obtained from the report of Korea Health Statistics 2015 published by the Korea Centers for Disease Control and Prevention $^{(20)}$. The chronic health conditions of interest included obesity, hypercholesterolaemia and hypertriacylglycerolaemia. Obesity was defined as BMI $\geq 25.0 \mathrm{~kg} / \mathrm{m}^{2}$ among adults aged $\geq 19$ years, according to the WHO Asia-Pacific region criteria $^{(21)}$. Hypercholesterolaemia and hypertriacylglycerolaemia were diagnosed based on the National Cholesterol Education Program Adult Treatment Panel III criteria ${ }^{(22)}$. Hypercholesterolaemia was defined as total cholesterol $\geq 240 \mathrm{mg} / \mathrm{dl}$ or the use of medication among adults aged $\geq 30$ years. Hypertriacylglycerolaemia was defined as fasting TAG $\geq 200 \mathrm{mg} / \mathrm{dl}$ among adults aged $\geq 30$ years. To adjust for differences in the age structure of each year survey, the age-standardized prevalence of chronic health conditions was presented using sex- and age-specific structures of the estimated population based on the 2005 Korea Census from 2007 through 2015 by sex. 


\section{Statistical analyses}

All statistical analyses were conducted using the statistical software package SAS version 9.4. All analyses accounted for the complex sampling design effect and used appropriate sampling weights, using the PROC SURVEY in the SAS program to produce estimates of the entire Korean adult population from the representative survey sample. Dietary variables were expressed as means with se by sex and age groups. Linear trends in intakes of total fat and fatty acids across the survey periods were compared using the generalized linear model after adjusting for sex, age, region and household income, where applicable.

\section{Results}

\section{Trends in intakes of energy and macronutrients}

Total energy and macronutrient intakes from 2007 through 2015 are summarized by sex in Table 1 . In both sexes, from 2007 to 2015, the total energy intake and percentage of energy from fat increased, whereas the percentage of energy from carbohydrate decreased from 2007 through 2015.

\section{Trends in intakes of total fat and fatty acids}

Trends in intakes of total fat and fatty acids across survey periods are presented by sex and age groups in Tables 2, 3 and 4 . In all sex and age groups, total fat intake ( $\mathrm{g}$ and $\% \mathrm{E})$ increased significantly from 2007 to 2015 ( $P<0.05)$. In addition, all sex and age groups showed significant increases in SFA intake: from $11.8 \mathrm{~g}(5.0 \% \mathrm{E})$ in 2007-2009 to $14.0 \mathrm{~g}(5.5 \% \mathrm{E})$ in $2013-2015 \mathrm{among}$ men and from $7.8 \mathrm{~g}$ $(4.5 \%)$ in $2007-2009$ to $10.0 \mathrm{~g}(5 \cdot 2 \% \mathrm{E})$ in $2013-2015$ among women. MUFA intake increased from 9.9 to $13.3 \mathrm{~g}$ $(P<0 \cdot 0001)$ from 2007 through 2015 , as did the proportion of energy from MUFA from 4.6 to $5.8 \% \mathrm{E}(P<0 \cdot 0001)$. PUFA intake ( $\mathrm{g}$ as well as \%E) also significantly increased in all sex and age groups except for young adults aged 1929 years. The intake of $n-6$ fatty acids increased in most sex and age groups, while the intake of $n-3$ fatty acids increased significantly only in adults aged $\geq 50$ years.

\section{Trends in prevalence of obesity and dyslipidaemia} Trends in prevalence of chronic health conditions from 2007 through 2015 are summarized by sex in Fig. 1. The prevalence of obesity among Korean adults increased from $31.7 \%$ in 2007 to $33.2 \%$ in 2015 . This increased rate was prominent only in men, with an increase from $36 \cdot 2 \%$ in 2007 to $39.7 \%$ in 2015 , while a subtle change was seen in women from $26.3 \%$ in 2007 to $26.0 \%$ in 2015 . However, the prevalence of hypercholesterolaemia increased gradually from 2007 through 2015 in both men (from 9.3 to $16.5 \%$ ) and women (from 11.6 to $19 \cdot 1 \%$ ). The prevalence of hypertriacylglycerolaemia changed with fluctuations from 2007 to 2015 in both men and women.

\section{Discussion}

Popkin suggested early that a sequence of major shifts in human diet and nutritional status in several phases typical in food use corresponded to the changes observed in the prevalence of nutrition-related diseases ${ }^{(23,24)}$. The author also noted that dietary patterns have converged towards diets high in saturated fat, sugar and refined foods of low

Table 1 Energy and macronutrient intakes in Korean adults from 2007 to 2015 by sex*. Data from the fourth (2007-2009), fifth (2010-2012) and sixth (2013-2015) Korea National Health and Nutrition Examination Survey

\begin{tabular}{|c|c|c|c|c|c|c|c|}
\hline & \multicolumn{2}{|c|}{ 2007-2009 } & \multicolumn{2}{|c|}{ 2010-2012 } & \multicolumn{2}{|c|}{ 2013-2015 } & \multirow[b]{2}{*}{$P$ trend $\dagger$} \\
\hline & Mean & SE & Mean & SE & Mean & SE & \\
\hline \multicolumn{8}{|l|}{ Total ( $n$ 47749) } \\
\hline Total energy (kJ) & 7991.4 & $39 \cdot 7$ & $8536 \cdot 6$ & $42 \cdot 7$ & 8643.7 & $40 \cdot 6$ & $<0.0001$ \\
\hline Total energy (kcal) & $1910 \cdot 0$ & 9.5 & $2040 \cdot 3$ & $10 \cdot 2$ & $2065 \cdot 9$ & $9 \cdot 7$ & $<0.0001$ \\
\hline Carbohydrate (\%E) & $67 \cdot 7$ & 0.2 & $66 \cdot 7$ & 0.2 & 65.6 & 0.1 & $<0.0001$ \\
\hline Protein (\%E) & 14.8 & 0.1 & $14 \cdot 8$ & 0.1 & $14 \cdot 6$ & 0.1 & 0.0856 \\
\hline Fat $(\% \mathrm{E})$ & $17 \cdot 6$ & 0.1 & $18 \cdot 6$ & 0.1 & $19 \cdot 8$ & 0.1 & $<0.0001$ \\
\hline \multicolumn{8}{|l|}{ Male $(n 20024)$} \\
\hline Total energy (kJ) & $9392 \cdot 2$ & $59 \cdot 4$ & 10074.7 & $61 \cdot 1$ & $10109 \cdot 8$ & $59 \cdot 8$ & $<0.0001$ \\
\hline Total energy (kcal) & 2244.8 & $14 \cdot 2$ & $2407 \cdot 9$ & $14 \cdot 6$ & $2416 \cdot 3$ & $14 \cdot 3$ & $<0.0001$ \\
\hline Carbohydrate (\%E) & 65.9 & 0.2 & 65.0 & 0.2 & 64.2 & 0.2 & $<0.0001$ \\
\hline Protein $(\% \mathrm{E})$ & $15 \cdot 4$ & 0.1 & $15 \cdot 4$ & 0.1 & $15 \cdot 2$ & $0 \cdot 1$ & 0.0649 \\
\hline Fat $(\% \mathrm{E})$ & $18 \cdot 7$ & 0.2 & $19 \cdot 6$ & 0.2 & $20 \cdot 6$ & 0.1 & $<0.0001$ \\
\hline \multicolumn{8}{|l|}{ Female (n 27 725) } \\
\hline Total energy $(\mathrm{kJ})$ & $6569 \cdot 7$ & $33 \cdot 1$ & 6978.9 & $36 \cdot 8$ & $7152 \cdot 1$ & $35 \cdot 1$ & $<0.0001$ \\
\hline Total energy (kcal) & $1570 \cdot 2$ & 7.9 & 1668.0 & $8 \cdot 8$ & $1709 \cdot 4$ & 8.4 & $<0.0001$ \\
\hline Carbohydrate (\%E) & 69.4 & 0.2 & 68.3 & 0.2 & $67 \cdot 0$ & 0.2 & $<0.0001$ \\
\hline Protein $(\% \mathrm{E})$ & $14 \cdot 1$ & 0.1 & $14 \cdot 2$ & 0.1 & $14 \cdot 1$ & 0.1 & 0.4340 \\
\hline Fat $(\% \mathrm{E})$ & $16 \cdot 5$ & 0.1 & 17.5 & $0 \cdot 1$ & $19 \cdot 0$ & $0 \cdot 1$ & $<0.0001$ \\
\hline
\end{tabular}

$\% \mathrm{E}$, percentage of energy.

${ }^{*}$ All statistical analyses accounted for the complex sampling design effect and appropriate sampling weights.

†Unadjusted. 
Table 2 Total fat intake (grams and percentage of energy (\%E)) in Korean adults from 2007 to 2015 by sex and age group*. Data from the fourth (2007-2009), fifth (2010-2012) and sixth (20132015) Korea National Health and Nutrition Examination Survey

\begin{tabular}{|c|c|c|c|c|c|c|c|c|c|c|c|c|c|c|}
\hline & \multicolumn{7}{|c|}{ Total fat $(\mathrm{g})$} & \multicolumn{7}{|c|}{ Total fat (\%E) } \\
\hline & \multicolumn{2}{|c|}{ 2007-2009 } & \multicolumn{2}{|c|}{ 2010-2012 } & \multicolumn{2}{|c|}{ 2013-2015 } & \multirow[b]{2}{*}{$P$ trend $\dagger$} & \multicolumn{2}{|c|}{ 2007-2009 } & \multicolumn{2}{|c|}{ 2010-2012 } & \multicolumn{2}{|c|}{ 2013-2015 } & \multirow[b]{2}{*}{$P$ trend $\dagger$} \\
\hline & Mean & $\mathrm{SE}$ & Mean & $\mathrm{SE}$ & Mean & $\mathrm{SE}$ & & Mean & SE & Mean & $\mathrm{SE}$ & Mean & $\mathrm{SE}$ & \\
\hline Total & 33.7 & 0.3 & 39.6 & 0.3 & $42 \cdot 2$ & 0.3 & $<0.0001$ & $16 \cdot 0$ & 0.1 & $17 \cdot 3$ & 0.1 & $18 \cdot 7$ & 0.1 & $<0.0001$ \\
\hline \multicolumn{15}{|l|}{ Sex } \\
\hline Male & $40 \cdot 2$ & 0.5 & $47 \cdot 2$ & 0.5 & $49 \cdot 0$ & 0.5 & $<0.0001$ & $16 \cdot 9$ & 0.1 & $18 \cdot 1$ & 0.1 & $19 \cdot 2$ & 0.1 & $<0.0001$ \\
\hline Female & $26 \cdot 8$ & 0.3 & 31.6 & 0.3 & $35 \cdot 1$ & 0.3 & $<0.0001$ & $15 \cdot 2$ & 0.1 & $16 \cdot 5$ & 0.1 & $18 \cdot 2$ & 0.1 & $<0.0001$ \\
\hline \multicolumn{15}{|l|}{ Age } \\
\hline $19-29$ years & 48.9 & 0.9 & 57.9 & $1 \cdot 1$ & 58.7 & 0.9 & $<0.0001$ & $22 \cdot 6$ & 0.2 & 24.4 & 0.3 & $25 \cdot 0$ & 0.2 & $<0.0001$ \\
\hline $30-49$ years & 41.5 & 0.5 & $47 \cdot 8$ & 0.6 & $50 \cdot 8$ & 0.5 & $<0.0001$ & $18 \cdot 7$ & 0.1 & $20 \cdot 1$ & 0.1 & 21.8 & 0.2 & $<0.0001$ \\
\hline $50-64$ years & $30 \cdot 8$ & 0.5 & $35 \cdot 3$ & 0.5 & $39 \cdot 3$ & 0.6 & $<0.0001$ & $14 \cdot 6$ & 0.2 & $15 \cdot 8$ & 0.2 & 17.4 & 0.2 & $<0.0001$ \\
\hline$\geq 65$ years & $21 \cdot 0$ & 0.4 & $23 \cdot 2$ & 0.4 & $25 \cdot 2$ & 0.4 & $<0.0001$ & 11.6 & 0.2 & 11.8 & 0.1 & $13 \cdot 0$ & 0.2 & $<0.0001$ \\
\hline \multicolumn{15}{|l|}{ Sex $\times$ age } \\
\hline Male, $19-29$ years & $57 \cdot 6$ & 1.5 & 67.3 & 1.7 & 67.9 & 1.5 & $<0.0001$ & $22 \cdot 9$ & 0.3 & 24.4 & 0.4 & $25 \cdot 3$ & 0.3 & $<0.0001$ \\
\hline Male, $30-49$ years & $50 \cdot 4$ & 0.7 & 57.7 & 0.9 & $59 \cdot 3$ & 0.8 & $<0.0001$ & $19 \cdot 8$ & 0.2 & 20.9 & 0.2 & $22 \cdot 2$ & 0.2 & $<0.0001$ \\
\hline Male, 50-64 years & $36 \cdot 9$ & 0.7 & $42 \cdot 1$ & 0.8 & $45 \cdot 4$ & 0.9 & $<0.0001$ & $15 \cdot 6$ & 0.2 & $16 \cdot 8$ & 0.2 & 17.9 & 0.2 & $<0.0001$ \\
\hline Male, $\geq 65$ years & $25 \cdot 1$ & 0.7 & 29.0 & 0.6 & $30 \cdot 2$ & 0.6 & $<0.0001$ & $12 \cdot 4$ & 0.2 & $13 \cdot 1$ & 0.2 & $14 \cdot 0$ & 0.2 & $<0.0001$ \\
\hline Female, $19-29$ years & $40 \cdot 2$ & 0.8 & 48.4 & $1 \cdot 1$ & 49.6 & 1.0 & $<0.0001$ & $22 \cdot 3$ & 0.3 & 24.4 & 0.4 & 24.6 & 0.3 & $<0.0001$ \\
\hline Female, $30-49$ years & 32.6 & 0.4 & 37.9 & 0.5 & $42 \cdot 3$ & 0.6 & $<0.0001$ & $17 \cdot 7$ & 0.2 & 19.2 & 0.2 & 21.4 & 0.2 & $<0.0001$ \\
\hline Female, $50-64$ years & $24 \cdot 8$ & 0.5 & $28 \cdot 6$ & 0.5 & $33 \cdot 1$ & 0.5 & $<0.0001$ & $13 \cdot 6$ & 0.2 & $14 \cdot 8$ & 0.2 & $16 \cdot 9$ & 0.2 & $<0.0001$ \\
\hline Female, $\geq 65$ years & $16 \cdot 7$ & 0.4 & $17 \cdot 6$ & 0.4 & $20 \cdot 1$ & 0.4 & $<0.0001$ & $10 \cdot 6$ & 0.2 & $10 \cdot 6$ & 0.2 & $12 \cdot 0$ & 0.2 & $<0.0001$ \\
\hline
\end{tabular}

${ }^{*}$ All statistical analyses accounted for the complex sampling design effect and appropriate sampling weights.

†Linear trends in total fat intake across the survey periods were compared using the generalized linear model after adjustment for sex, age, region and household income, where applicable. 


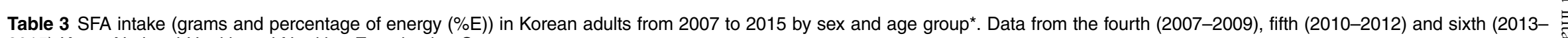
2015) Korea National Health and Nutrition Examination Survey

\begin{tabular}{|c|c|c|c|c|c|c|c|c|c|c|c|c|c|c|}
\hline & \multicolumn{7}{|c|}{ SFA (g) } & \multicolumn{7}{|c|}{ SFA (\%E) } \\
\hline & \multicolumn{2}{|c|}{ 2007-2009 } & \multicolumn{2}{|c|}{ 2010-2012 } & \multicolumn{2}{|c|}{ 2013-2015 } & \multirow[b]{2}{*}{$P$ trend $†$} & \multicolumn{2}{|c|}{ 2007-2009 } & \multicolumn{2}{|c|}{ 2010-2012 } & \multicolumn{2}{|c|}{ 2013-2015 } & \multirow[b]{2}{*}{$P$ trend $\dagger$} \\
\hline & Mean & SE & Mean & SE & Mean & SE & & Mean & SE & Mean & SE & Mean & SE & \\
\hline Total & 9.9 & 0.1 & $11 \cdot 7$ & 0.1 & $12 \cdot 0$ & 0.1 & $<0.0001$ & 4.7 & 0.03 & $5 \cdot 1$ & 0.04 & $5 \cdot 3$ & 0.03 & $<0.0001$ \\
\hline \multicolumn{15}{|l|}{ Sex } \\
\hline Male & $11 \cdot 8$ & 0.2 & $14 \cdot 0$ & 0.2 & $14 \cdot 0$ & 0.2 & $<0.0001$ & $5 \cdot 0$ & 0.05 & 5.4 & 0.05 & 5.5 & 0.05 & $<0.0001$ \\
\hline Female & $7 \cdot 8$ & 0.1 & 9.4 & 0.1 & $10 \cdot 0$ & 0.1 & $<0.0001$ & 4.5 & 0.04 & 4.9 & 0.04 & $5 \cdot 2$ & 0.04 & $<0.0001$ \\
\hline \multicolumn{15}{|l|}{ Age } \\
\hline 19-29 years & 14.9 & 0.3 & $17 \cdot 7$ & 0.4 & $17 \cdot 7$ & 0.3 & $<0.0001$ & $6 \cdot 9$ & 0.1 & 7.5 & $0 \cdot 1$ & $7 \cdot 6$ & 0.1 & $<0.0001$ \\
\hline $30-49$ years & $12 \cdot 3$ & 0.2 & $14 \cdot 3$ & 0.2 & 14.7 & 0.2 & $<0.0001$ & $5 \cdot 6$ & 0.1 & $6 \cdot 0$ & 0.1 & $6 \cdot 3$ & 0.1 & $<0.0001$ \\
\hline 50-64 years & 8.9 & 0.1 & $10 \cdot 2$ & 0.2 & $10 \cdot 8$ & 0.2 & $<0.0001$ & $4 \cdot 2$ & 0.1 & 4.6 & 0.1 & $4 \cdot 8$ & 0.1 & $<0.0001$ \\
\hline$\geq 65$ years & $6 \cdot 1$ & 0.1 & $6 \cdot 8$ & $0 \cdot 1$ & $7 \cdot 0$ & 0.1 & $<0.0001$ & $3 \cdot 3$ & $0 \cdot 1$ & 3.5 & 0.1 & $3 \cdot 6$ & 0.1 & 0.0002 \\
\hline \multicolumn{15}{|l|}{ Sex $\times$ age } \\
\hline Male, $19-29$ years & $17 \cdot 7$ & 0.5 & $20 \cdot 4$ & 0.6 & $20 \cdot 3$ & 0.5 & 0.0006 & $7 \cdot 0$ & 0.1 & $7 \cdot 4$ & 0.2 & $7 \cdot 6$ & 0.1 & 0.0016 \\
\hline Male, 30-49 years & 14.9 & 0.2 & $17 \cdot 3$ & $0 \cdot 3$ & $17 \cdot 3$ & 0.3 & $<0.0001$ & $5 \cdot 9$ & $0 \cdot 1$ & $6 \cdot 3$ & 0.1 & $6 \cdot 5$ & 0.1 & $<0.0001$ \\
\hline Male, 50-64 years & $10 \cdot 7$ & 0.2 & $12 \cdot 3$ & 0.2 & $12 \cdot 7$ & 0.3 & $<0.0001$ & 4.5 & $0 \cdot 1$ & 4.9 & 0.1 & $5 \cdot 0$ & 0.1 & $<0.0001$ \\
\hline Male, $\geq 65$ years & $7 \cdot 3$ & 0.2 & 8.5 & 0.2 & 8.3 & 0.2 & 0.0011 & 3.6 & $0 \cdot 1$ & 3.9 & 0.1 & 3.9 & 0.1 & 0.0227 \\
\hline Female, 19-29 years & $12 \cdot 2$ & 0.3 & $15 \cdot 1$ & 0.4 & $15 \cdot 1$ & 0.3 & $<0.0001$ & $6 \cdot 8$ & $0 \cdot 1$ & $7 \cdot 6$ & 0.1 & 7.5 & $0 \cdot 1$ & 0.0002 \\
\hline Female, 30-49 years & 9.7 & 0.1 & $11 \cdot 3$ & 0.2 & $12 \cdot 2$ & 0.2 & $<0.0001$ & $5 \cdot 2$ & $0 \cdot 1$ & $5 \cdot 8$ & 0.1 & $6 \cdot 2$ & $0 \cdot 1$ & $<0.0001$ \\
\hline Female, $50-64$ years & $7 \cdot 1$ & 0.1 & $8 \cdot 1$ & 0.1 & 8.9 & 0.2 & $<0.0001$ & 3.9 & $0 \cdot 1$ & 4.3 & 0.1 & 4.6 & 0.1 & $<0.0001$ \\
\hline Female, $\geq 65$ years & 4.7 & 0.1 & $5 \cdot 1$ & $0 \cdot 1$ & $5 \cdot 5$ & 0.1 & $<0.0001$ & $3 \cdot 1$ & $0 \cdot 1$ & $3 \cdot 1$ & 0.1 & $3 \cdot 3$ & $0 \cdot 1$ & 0.0004 \\
\hline
\end{tabular}

${ }^{*}$ All statistical analyses accounted for the complex sampling design effect and appropriate sampling weights.

tLinear trends in SFA intake across the survey periods were compared using the generalized linear model after adjustment for sex, age, region and household income, where applicable. 


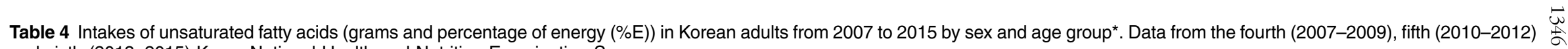
and sixth (2013-2015) Korea National Health and Nutrition Examination Survey

\begin{tabular}{|c|c|c|c|c|c|c|c|c|c|c|c|c|c|c|}
\hline & \multicolumn{7}{|c|}{ MUFA (g) } & \multicolumn{7}{|c|}{ MUFA (\%E) } \\
\hline & \multicolumn{2}{|c|}{ 2007-2009 } & \multicolumn{2}{|c|}{ 2010-2012 } & \multicolumn{2}{|c|}{ 2013-2015 } & \multirow[b]{2}{*}{$P$ trend $\dagger$} & \multicolumn{2}{|c|}{ 2007-2009 } & \multicolumn{2}{|c|}{ 2010-2012 } & \multicolumn{2}{|c|}{ 2013-2015 } & \multirow[b]{2}{*}{$P$ trend $\dagger$} \\
\hline & Mean & SE & Mean & SE & Mean & SE & & Mean & SE & Mean & SE & Mean & SE & \\
\hline Total & 9.9 & $0 \cdot 1$ & $12 \cdot 0$ & $0 \cdot 1$ & $13 \cdot 3$ & $0 \cdot 1$ & $<0.0001$ & $4 \cdot 6$ & 0.04 & $5 \cdot 1$ & 0.04 & $5 \cdot 8$ & 0.04 & $<0.0001$ \\
\hline \multicolumn{15}{|l|}{ Sex } \\
\hline Male & 11.9 & 0.2 & $14 \cdot 4$ & 0.2 & $15 \cdot 5$ & 0.2 & $<0.0001$ & 4.9 & 0.1 & 5.4 & 0.1 & $6 \cdot 0$ & 0.1 & $<0.0001$ \\
\hline Female & $7 \cdot 7$ & 0.1 & $9 \cdot 4$ & $0 \cdot 1$ & $10 \cdot 9$ & $0 \cdot 1$ & $<0.0001$ & $4 \cdot 3$ & 0.04 & $4 \cdot 8$ & 0.04 & $5 \cdot 6$ & 0.04 & $<0.0001$ \\
\hline \multicolumn{15}{|l|}{ Age } \\
\hline 19-29 years & $15 \cdot 3$ & 0.3 & $18 \cdot 5$ & 0.4 & $19 \cdot 1$ & 0.4 & $<0.0001$ & $7 \cdot 0$ & $0 \cdot 1$ & $7 \cdot 7$ & 0.1 & $8 \cdot 1$ & 0.1 & $<0.0001$ \\
\hline $30-49$ years & 12.5 & 0.2 & $14 \cdot 7$ & 0.2 & $16 \cdot 3$ & 0.2 & $<0.0001$ & $5 \cdot 6$ & 0.1 & $6 \cdot 1$ & 0.1 & 6.9 & 0.1 & $<0.0001$ \\
\hline 50-64 years & $8 \cdot 8$ & 0.2 & $10 \cdot 4$ & 0.2 & $12 \cdot 1$ & 0.2 & $<0.0001$ & $4 \cdot 1$ & $0 \cdot 1$ & $4 \cdot 6$ & 0.1 & $5 \cdot 3$ & 0.1 & $<0.0001$ \\
\hline$\geq 65$ years & $5 \cdot 8$ & 0.1 & 6.5 & $0 \cdot 1$ & 7.4 & $0 \cdot 1$ & $<0.0001$ & $3 \cdot 1$ & 0.1 & 3.3 & 0.1 & 3.8 & 0.1 & $<0.0001$ \\
\hline \multicolumn{15}{|l|}{ Sex $\times$ age } \\
\hline Male, 19-29 years & $18 \cdot 2$ & 0.5 & $21 \cdot 6$ & 0.6 & $22 \cdot 4$ & 0.6 & $<0.0001$ & $7 \cdot 2$ & $0 \cdot 1$ & $7 \cdot 8$ & 0.2 & $8 \cdot 3$ & 0.2 & $<0.0001$ \\
\hline Male, $30-49$ years & $15 \cdot 3$ & 0.3 & $18 \cdot 0$ & 0.4 & $19 \cdot 1$ & 0.3 & $<0.0001$ & 5.9 & $0 \cdot 1$ & $6 \cdot 4$ & 0.1 & $7 \cdot 1$ & 0.1 & $<0.0001$ \\
\hline Male, $50-64$ years & $10 \cdot 7$ & 0.3 & 12.5 & 0.3 & $14 \cdot 2$ & 0.3 & $<0.0001$ & 4.5 & $0 \cdot 1$ & 4.9 & 0.1 & 5.5 & 0.1 & $<0.0001$ \\
\hline Male, $\geq 65$ years & $7 \cdot 0$ & 0.2 & $8 \cdot 3$ & 0.2 & $9 \cdot 0$ & 0.2 & $<0.0001$ & $3 \cdot 4$ & $0 \cdot 1$ & $3 \cdot 7$ & 0.1 & $4 \cdot 1$ & 0.1 & $<0.0001$ \\
\hline Female, $19-29$ years & $12 \cdot 4$ & 0.3 & $15 \cdot 4$ & 0.4 & $15 \cdot 8$ & 0.4 & $<0.0001$ & $6 \cdot 8$ & $0 \cdot 1$ & $7 \cdot 7$ & 0.1 & $7 \cdot 8$ & 0.1 & $<0.0001$ \\
\hline Female, $30-49$ years & $9 \cdot 7$ & 0.1 & 11.4 & 0.2 & 13.5 & 0.2 & $<0.0001$ & $5 \cdot 2$ & 0.1 & 5.8 & 0.1 & $6 \cdot 8$ & 0.1 & $<0.0001$ \\
\hline Female, 50-64 years & $6 \cdot 9$ & 0.2 & $8 \cdot 2$ & 0.2 & $10 \cdot 0$ & 0.2 & $<0.0001$ & $3 \cdot 8$ & $0 \cdot 1$ & $4 \cdot 2$ & 0.1 & $5 \cdot 1$ & 0.1 & $<0.0001$ \\
\hline \multirow[t]{4}{*}{ Female, $\geq 65$ years } & 4.4 & $0 \cdot 1$ & $4 \cdot 8$ & $0 \cdot 1$ & $5 \cdot 7$ & $0 \cdot 1$ & $<0.0001$ & $2 \cdot 8$ & 0.1 & $2 \cdot 8$ & $0 \cdot 1$ & $3 \cdot 4$ & $0 \cdot 1$ & $<0.0001$ \\
\hline & \multicolumn{7}{|c|}{ PUFA (g) } & \multicolumn{7}{|c|}{ PUFA (\%E) } \\
\hline & \multicolumn{2}{|c|}{ 2007-2009 } & \multicolumn{2}{|c|}{ 2010-2012 } & \multicolumn{2}{|c|}{ 2013-2015 } & & \multicolumn{2}{|c|}{ 2007-2009 } & \multicolumn{2}{|c|}{ 2010-2012 } & 201 & 15 & \\
\hline & Mean & SE & Mean & SE & Mean & SE & $P$ trend $\dagger$ & Mean & SE & Mean & SE & Mean & SE & $P$ trend $†$ \\
\hline Total & $8 \cdot 7$ & 0.1 & $9 \cdot 8$ & $0 \cdot 1$ & $10 \cdot 7$ & $0 \cdot 1$ & $<0.0001$ & 4.2 & 0.03 & $4 \cdot 3$ & 0.03 & 4.7 & 0.03 & $<0.0001$ \\
\hline Sex & & & & & & & & & & & & & & \\
\hline Male & $10 \cdot 3$ & $0 \cdot 1$ & 11.6 & $0 \cdot 1$ & $12 \cdot 2$ & 0.1 & $<0.0001$ & 4.4 & 0.04 & 4.5 & 0.04 & 4.8 & 0.04 & $<0.0001$ \\
\hline Female & $7 \cdot 1$ & 0.1 & 7.9 & $0 \cdot 1$ & $9 \cdot 0$ & $0 \cdot 1$ & $<0.0001$ & $4 \cdot 0$ & 0.04 & $4 \cdot 1$ & 0.04 & 4.7 & 0.04 & $<0.0001$ \\
\hline Age & & & & & & & & & & & & & & \\
\hline 19-29 years & $12 \cdot 2$ & 0.3 & $13 \cdot 7$ & 0.3 & $13 \cdot 9$ & 0.3 & $<0.0001$ & $5 \cdot 7$ & 0.09 & $5 \cdot 8$ & 0.09 & $5 \cdot 9$ & 0.09 & 0.0649 \\
\hline 30-49 years & $10 \cdot 4$ & 0.1 & $11 \cdot 6$ & 0.2 & $12 \cdot 4$ & 0.2 & $<0.0001$ & 4.7 & 0.04 & 4.9 & 0.05 & $5 \cdot 3$ & 0.05 & $<0.0001$ \\
\hline 50-64 years & $8 \cdot 1$ & 0.1 & $9 \cdot 0$ & $0 \cdot 1$ & $10 \cdot 5$ & 0.2 & $<0.0001$ & 3.9 & 0.05 & 4.0 & 0.05 & 4.7 & 0.05 & $<0.0001$ \\
\hline$\geq 65$ years & $5 \cdot 7$ & 0.1 & $6 \cdot 1$ & $0 \cdot 1$ & 6.9 & $0 \cdot 1$ & $<0.0001$ & $3 \cdot 1$ & 0.05 & $3 \cdot 1$ & 0.04 & 3.6 & 0.05 & $<0.0001$ \\
\hline Sex $\times$ age & & & & & & & & & & & & & & \\
\hline Male, 19-29 years & $14 \cdot 2$ & 0.4 & $16 \cdot 2$ & 0.5 & $16 \cdot 1$ & 0.4 & 0.0018 & $5 \cdot 7$ & 0.11 & $5 \cdot 9$ & 0.13 & $6 \cdot 0$ & 0.12 & 0.0734 \\
\hline Male, $30-49$ years & $12 \cdot 7$ & 0.2 & $13 \cdot 9$ & 0.2 & 14.5 & 0.2 & $<0.0001$ & $5 \cdot 0$ & 0.06 & $5 \cdot 1$ & 0.06 & $5 \cdot 4$ & 0.06 & $<0.0001$ \\
\hline Male, $50-64$ years & 9.5 & 0.2 & 10.5 & 0.2 & 11.8 & 0.2 & $<0.0001$ & $4 \cdot 1$ & 0.07 & 4.2 & 0.06 & $4 \cdot 7$ & 0.07 & $<0.0001$ \\
\hline Male, $\geq 65$ years & $6 \cdot 6$ & 0.2 & $7 \cdot 3$ & 0.2 & $8 \cdot 1$ & $0 \cdot 2$ & $<0.0001$ & $3 \cdot 3$ & 0.07 & $3 \cdot 3$ & 0.06 & $3 \cdot 8$ & 0.07 & $<0.0001$ \\
\hline Female, $19-29$ years & $10 \cdot 2$ & 0.3 & $11 \cdot 2$ & 0.3 & $11 \cdot 8$ & 0.3 & 0.0003 & $5 \cdot 7$ & 0.12 & 5.7 & 0.11 & $5 \cdot 8$ & 0.12 & 0.3690 \\
\hline Female, $30-49$ years & $8 \cdot 2$ & 0.1 & $9 \cdot 3$ & 0.2 & $10 \cdot 4$ & 0.2 & $<0.0001$ & 4.5 & 0.05 & 4.7 & 0.06 & $5 \cdot 2$ & 0.06 & $<0.0001$ \\
\hline Female, 50-64 years & $6 \cdot 7$ & 0.1 & 7.5 & $0 \cdot 1$ & $9 \cdot 2$ & 0.2 & $<0.0001$ & $3 \cdot 7$ & 0.06 & 3.9 & 0.06 & 4.7 & 0.07 & $<0.0001$ \\
\hline Female, $\geq 65$ years & 4.6 & 0.1 & 4.8 & $0 \cdot 1$ & $5 \cdot 6$ & $0 \cdot 1$ & $<0.0001$ & 3.0 & 0.06 & $2 \cdot 9$ & 0.05 & 3.4 & 0.06 & $<0.0001$ \\
\hline
\end{tabular}




\begin{tabular}{|c|c|c|c|c|c|c|c|c|c|c|c|c|c|c|}
\hline & \multicolumn{7}{|c|}{$n-3$ FA (g) } & \multicolumn{7}{|c|}{$n-3$ FA (\%E) } \\
\hline & \multicolumn{2}{|c|}{ 2007-2009 } & \multicolumn{2}{|c|}{ 2010-2012 } & \multicolumn{2}{|c|}{ 2013-2015 } & \multirow[b]{2}{*}{$P$ trend $†$} & \multicolumn{2}{|c|}{ 2007-2009 } & \multicolumn{2}{|c|}{ 2010-2012 } & \multicolumn{2}{|c|}{ 2013-2015 } & \multirow[b]{2}{*}{$P$ trend $\dagger$} \\
\hline & Mean & SE & Mean & SE & Mean & SE & & Mean & SE & Mean & SE & Mean & SE & \\
\hline Total & 1.40 & 0.02 & 1.52 & 0.02 & 1.57 & 0.02 & $<0.0001$ & 0.68 & 0.01 & 0.68 & 0.01 & 0.72 & 0.01 & 0.0001 \\
\hline \multicolumn{15}{|l|}{ Sex } \\
\hline Male & 1.65 & 0.03 & 1.78 & 0.03 & 1.79 & 0.03 & 0.0003 & 0.71 & 0.01 & 0.71 & 0.01 & 0.72 & 0.01 & 0.2046 \\
\hline Female & $1 \cdot 14$ & 0.02 & 1.26 & 0.02 & 1.35 & 0.02 & $<0.0001$ & 0.65 & 0.01 & 0.66 & 0.01 & $0 \cdot 71$ & 0.01 & $<0.0001$ \\
\hline \multicolumn{15}{|l|}{ Age } \\
\hline 19-29 years & 1.55 & 0.04 & 1.69 & 0.04 & 1.66 & 0.04 & 0.066 & 0.72 & 0.01 & 0.73 & 0.01 & 0.71 & 0.01 & 0.5538 \\
\hline $30-49$ years & 1.56 & 0.02 & 1.71 & 0.03 & 1.67 & 0.03 & 0.0025 & 0.72 & 0.01 & 0.73 & 0.01 & 0.73 & 0.01 & 0.3487 \\
\hline 50-64 years & 1.41 & 0.03 & 1.52 & 0.03 & 1.72 & 0.04 & $<0.0001$ & 0.69 & 0.01 & 0.69 & 0.01 & 0.78 & 0.01 & $<0.0001$ \\
\hline$\geq 65$ years & 1.05 & 0.03 & 1.09 & 0.03 & 1.23 & 0.03 & $<0.0001$ & 0.59 & 0.01 & 0.56 & 0.01 & 0.64 & 0.01 & 0.0017 \\
\hline \multicolumn{15}{|l|}{ Sex $\times$ age } \\
\hline Male, $19-29$ years & 1.80 & 0.07 & 1.98 & 0.07 & 1.92 & 0.06 & 0.2311 & 0.72 & 0.02 & 0.73 & 0.02 & 0.72 & 0.02 & 0.9339 \\
\hline Male, $30-49$ years & 1.86 & 0.04 & 1.99 & 0.04 & 1.92 & 0.04 & 0.2744 & 0.74 & 0.01 & 0.73 & 0.01 & 0.73 & 0.01 & 0.4865 \\
\hline Male, $50-64$ years & 1.64 & 0.04 & 1.76 & 0.05 & 1.89 & 0.05 & 0.0004 & 0.71 & 0.02 & 0.72 & 0.02 & 0.76 & 0.02 & 0.0447 \\
\hline Male, $\geq 65$ years & 1.22 & 0.04 & 1.31 & 0.04 & 1.44 & 0.04 & 0.0003 & 0.61 & 0.02 & 0.60 & 0.02 & 0.68 & 0.02 & 0.0037 \\
\hline Female, $19-29$ years & 1.30 & 0.04 & 1.40 & 0.04 & 1.41 & 0.05 & 0.0834 & 0.72 & 0.02 & 0.72 & 0.02 & 0.69 & 0.02 & 0.2575 \\
\hline Female, $30-49$ years & 1.25 & 0.02 & 1.43 & 0.03 & 1.42 & 0.03 & $<0.0001$ & 0.69 & 0.01 & 0.72 & 0.01 & 0.73 & 0.01 & 0.0224 \\
\hline Female, $50-64$ years & 1.18 & 0.03 & 1.28 & 0.03 & 1.55 & 0.04 & $<0.0001$ & 0.66 & 0.02 & 0.66 & 0.01 & 0.80 & 0.02 & $<0.0001$ \\
\hline Female, $\geq 65$ years & 0.87 & 0.03 & 0.88 & 0.03 & 1.02 & 0.03 & 0.0006 & 0.56 & 0.02 & 0.52 & 0.01 & 0.61 & 0.02 & 0.0299 \\
\hline
\end{tabular}

$n-6$ FA (g)

\begin{tabular}{|c|c|c|c|c|c|c|c|c|c|c|c|c|c|c|}
\hline & \multicolumn{2}{|c|}{ 2007-2009 } & \multicolumn{2}{|c|}{ 2010-2012 } & \multicolumn{2}{|c|}{ 2013-2015 } & \multirow[b]{2}{*}{$P$ trend $\dagger$} & \multicolumn{2}{|c|}{ 2007-2009 } & \multicolumn{2}{|c|}{ 2010-2012 } & \multicolumn{2}{|c|}{ 2013-2015 } & \multirow[b]{2}{*}{$P$ trend $\dagger$} \\
\hline & Mean & SE & Mean & SE & Mean & SE & & Mean & SE & Mean & SE & Mean & SE & \\
\hline Total & $7 \cdot 4$ & $0 \cdot 1$ & 8.4 & 0.1 & $9 \cdot 2$ & 0.1 & $<0.0001$ & 3.5 & 0.03 & 3.7 & 0.03 & $4 \cdot 1$ & 0.03 & $<0.0001$ \\
\hline \multicolumn{15}{|l|}{ Sex } \\
\hline Male & 8.8 & $0 \cdot 1$ & 9.9 & 0.1 & $10 \cdot 5$ & 0.1 & $<0.0001$ & $3 \cdot 7$ & 0.04 & 3.8 & 0.04 & 4.1 & 0.04 & $<0.0001$ \\
\hline Female & $6 \cdot 0$ & 0.1 & 6.7 & 0.1 & $7 \cdot 7$ & 0.1 & $<0.0001$ & 3.4 & 0.03 & 3.5 & 0.03 & 4.0 & 0.03 & $<0.0001$ \\
\hline \multicolumn{15}{|l|}{ Age } \\
\hline 19-29 years & $10 \cdot 7$ & 0.2 & $12 \cdot 2$ & 0.3 & $12 \cdot 4$ & 0.2 & $<0.0001$ & $5 \cdot 0$ & 0.08 & $5 \cdot 1$ & 0.08 & $5 \cdot 2$ & 0.08 & 0.0235 \\
\hline $30-49$ years & $9 \cdot 0$ & 0.1 & $10 \cdot 0$ & 0.1 & $10 \cdot 8$ & 0.1 & $<0.0001$ & 4.0 & 0.04 & $4 \cdot 2$ & 0.04 & 4.6 & 0.04 & $<0.0001$ \\
\hline $50-64$ years & 6.8 & 0.1 & $7 \cdot 6$ & 0.1 & 8.8 & 0.1 & $<0.0001$ & $3 \cdot 2$ & 0.04 & 3.4 & 0.04 & 3.9 & 0.05 & $<0.0001$ \\
\hline$\geq 65$ years & 4.6 & 0.1 & $5 \cdot 0$ & 0.1 & $5 \cdot 7$ & 0.1 & $<0.0001$ & $2 \cdot 6$ & 0.04 & $2 \cdot 5$ & 0.04 & $2 \cdot 9$ & 0.05 & $<0.0001$ \\
\hline \multicolumn{15}{|l|}{ Sex $\times$ age } \\
\hline Male, $19-29$ years & 12.5 & 0.4 & 14.4 & 0.4 & 14.4 & 0.4 & 0.0008 & 5.0 & 0.10 & $5 \cdot 2$ & 0.12 & $5 \cdot 3$ & 0.11 & 0.0392 \\
\hline Male, $30-49$ years & $10 \cdot 9$ & 0.2 & $12 \cdot 1$ & 0.2 & 12.6 & 0.2 & $<0.0001$ & 4.3 & 0.05 & 4.4 & 0.06 & 4.7 & 0.06 & $<0.0001$ \\
\hline Male, 50-64 years & 7.9 & 0.2 & $8 \cdot 8$ & 0.2 & $10 \cdot 0$ & 0.2 & $<0.0001$ & $3 \cdot 4$ & 0.06 & 3.5 & 0.06 & 4.0 & 0.06 & $<0.0001$ \\
\hline Male, $\geq 65$ years & $5 \cdot 4$ & 0.2 & $6 \cdot 1$ & 0.1 & $6 \cdot 8$ & 0.2 & $<0.0001$ & $2 \cdot 7$ & 0.06 & $2 \cdot 8$ & 0.05 & 3.1 & 0.06 & $<0.0001$ \\
\hline Female, $19-29$ years & 9.0 & 0.2 & 9.9 & 0.3 & 10.5 & 0.3 & $<0.0001$ & 5.0 & 0.11 & $5 \cdot 0$ & 0.10 & 5.1 & 0.10 & 0.2105 \\
\hline Female, 30-49 years & $7 \cdot 0$ & 0.1 & 7.9 & 0.1 & $9 \cdot 0$ & 0.1 & $<0.0001$ & 3.8 & 0.04 & 4.0 & 0.05 & 4.5 & 0.05 & $<0.0001$ \\
\hline Female, $50-64$ years & $5 \cdot 6$ & 0.1 & $6 \cdot 3$ & 0.1 & $7 \cdot 7$ & 0.1 & $<0.0001$ & $3 \cdot 1$ & 0.05 & $3 \cdot 2$ & 0.05 & 3.9 & 0.06 & $<0.0001$ \\
\hline Female, $\geq 65$ years & $3 \cdot 8$ & 0.1 & 3.9 & 0.1 & $4 \cdot 6$ & 0.1 & $<0.0001$ & $2 \cdot 4$ & 0.05 & $2 \cdot 3$ & 0.05 & $2 \cdot 8$ & 0.05 & $<0.0001$ \\
\hline
\end{tabular}

FA, fatty acids

†Linear trends in intakes of unsaturated fatty acids across the survey periods were compared using the generalized linear model after adjustment for sex, age, region and household income, where applicable. 
(a)

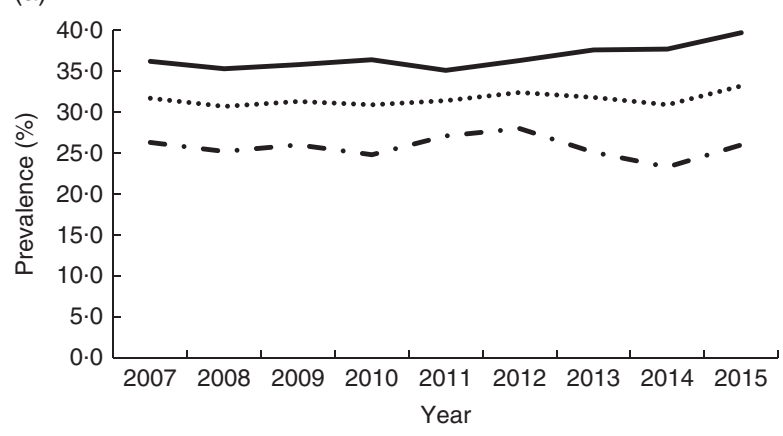

(b)

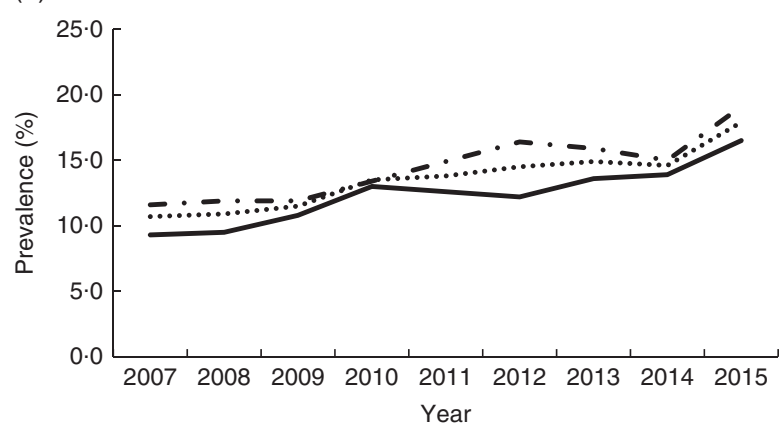

(c)

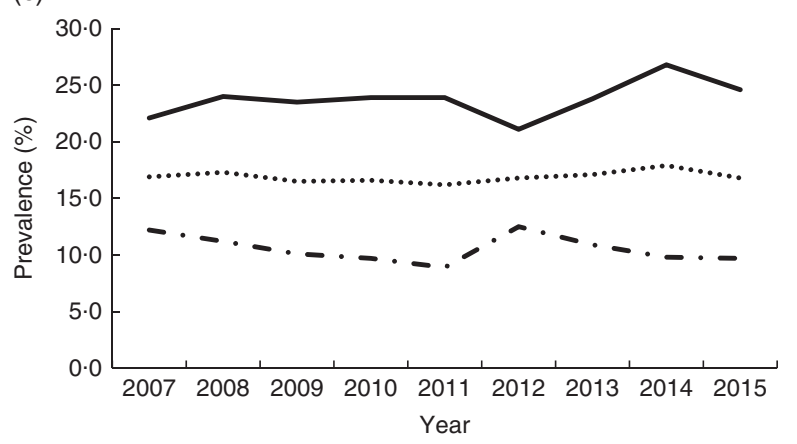

Fig. 1 Prevalence of chronic health conditions in Korean adults from 2007 to 2015 by sex (......, total; — male; - . - , female): (a) obesity; (b) hypercholesterolaemia; (c) hypertriacylglycerolaemia. Data from Korea Health Statistics 2015 report published by the Korea Centers for Disease Control and Prevention ${ }^{(20)}$

dietary fibre, leading to the increased prevalence of obesity and the related chronic degenerative diseases ${ }^{(23)}$.

Early studies on SFA and cardiovascular health indicated that dietary SFA and cholesterol intakes led to increases in CHD and hypercholesterolaemia ${ }^{(2,25)}$. Those points of evidence supported the recommendation of reducing the consumption of total fat and SFA in Western nations where fat intake is higher than in other parts of the world ${ }^{(26)}$. The consequential nutrition transitions in developed countries led to behavioural changes that reduced diet-related NCD with the decreased intakes of refined foods, meats and dairy products and the increased intakes of fruits, vegetables and complex carbohydrates ${ }^{(23)}$. A study using National Health and Nutrition Examination Survey data on trends in intake of energy and macronutrients intake from
1971-1974 through 1999-2000 showed a statistically significant decrease in intake of fat in the US population conforming to the dietary guideline ${ }^{(27)}$. Population energy and macronutrient intakes have remained relatively stable over the decades ${ }^{(28,29)}$. Although the prevalence of obesity is still increasing in the USA, the rate of increase has slowed in the most recent decade to about half of what it was two decades ago (22.9\% in 1988-1994, 30.4\% in 1999$2002,33 \cdot 4 \%$ in $2003-2006$ and $36.4 \%$ in $2011-2014)^{(29)}$.

Nevertheless, the general dietary recommendation on reducing fat has recently been challenged, as the inverse increase in carbohydrate intake has been suggested as a risk factor for $\mathrm{NCD}^{(26,30)}$. Recent reviews have indicated that there is no association between SFA intake and CHD. Further, a meta-analysis of prospective cohort studies showed no significant association of dietary SFA with an increased risk of CHD or CVD ${ }^{(10)}$. Several following systematic reviews of randomized controlled trials and prospective cohort studies showed no evidence for a reduction in CHD mortality with a reduction in dietary $\mathrm{fat}^{(4,5)}$. In the Hispanic population of Mexican origin, the percentage of energy from total fat and SFA decreased while that from carbohydrate increased between 19821984 and 1999-2006. Interestingly, over the same period, the prevalence of obesity and related illnesses, including type 2 diabetes, increased in the population ${ }^{(31)}$.

It is difficult to attribute health problems observed in a population simply to dietary changes, such as an increase in carbohydrate as a substitute for fat. The trends in energy intake of various populations show a steady increase and changes in SFA intake above the dietary guidelines parallel the changes in the prevalence of obesity and diet-related $\mathrm{NCD}^{(28,29)}$. The lack of any association of SFA with CVD after controlling for energy may suggest that the contributions of macronutrients are comparable to each other. Many epidemiological studies in which macronutrients were substituted for SFA found no associations between SFA and CHD risk ${ }^{(10)}$ : these studies did not specify what macronutrients were used as substitutes for SFA and did not distinguish between simple $v$. complex carbohydrates replacing $\mathrm{SFA}^{(9)}$. Since different types of carbohydrate result in different metabolic effects on blood sugar levels, carbohydrate type may be a stronger indicator of CVD risk than total carbohydrate ${ }^{(32,33)}$. In several population studies, it is common for SFA to be replaced by refined starches and/or added sugars ${ }^{(9)}$. Replacing dietary SFA with other types of fat, especially cis-PUFA, has been reported to reduce CVD risk in several randomized controlled trials ${ }^{(34,35)}$ and observational studies ${ }^{(36)}$. According to the majority of the literature, dietary saturated fats, compared with carbohydrates and unsaturated fats, raise plasma LDL-cholesterol, a causal risk factor for $\mathrm{CHD}^{(1,11,34-37)}$.

In addition, individual SFA affect plasma lipoprotein levels differently ${ }^{(11,37-39)}$ : myristic acid and palmitic acids have the greatest effect, while stearic acid has not shown 
any effect on elevating blood cholesterol. The most effective replacement for SFA in terms of CHD has been shown to be linoleic acid. Therefore, not only should the type of fat be considered, but also so should food sources which have specific fatty acid profiles. Pork, milk and instant noodles, which were the major source of total fat and SFA in the Korean population, have high myristic and palmitic acid contents $^{(15)}$. Low-fat dairy and the replacement of fat sources with oils, nuts and seeds should be promoted.

Nutrition transitions have been reported worldwide along with changes in demographics and economic transitions, but these changes have been seen substantially more in developing countries ${ }^{(23,40,41)}$. In the present study focusing on the Korean population, we found that the energy consumption of the Korean population increased gradually along with total fat consumption and the proportion of energy from fat. The increase of total fat intake is explained by increases in all of the components proportionally from the 2007-2009 to the 2013-2015 survey period $(P<0.05)$ : saturated (from 4.7 to $5.3 \% \mathrm{E}$ ), monounsaturated ( 4.6 to $5.8 \% \mathrm{E})$ and polyunsaturated ( 4.2 to $4.7 \% \mathrm{E})$. Traditionally, the Korean population has quite a low proportion of energy from fat. The observed nutrition transitions are the increase in consumption of SFA along with the increase in prevalence of hypercholesterolaemia. The proportion of energy from carbohydrate has traditionally been high in the Korean population with a high prevalence of hypertriacylglycerolaemia.

Therefore, public dietary recommendations need to be carefully developed based on unique situations driven by evidence on the proportion of energy from fat and carbohydrate. In order to address the emerging health problems related to dietary behaviours in specific populations, recommendations need to be specific but robust enough to consider global views of changing trends in diet and lifestyle in relation to health outcomes. Even though the Korean population consumes low energy percentage from SFA, researchers and policy makers need to be mindful of the changing trends. The types of carbohydrate and fat and their sources have to be considered simultaneously along with the total amount, in order to avoid unfavourable nutrition transitions while enabling regionally, culturally and nationally acceptable healthful behavioural changes in a timely manner.

\section{Conclusions}

Although the intakes of total fat and SFA are relatively low in Korea compared with those in Western countries, the public health messages in Korea have been very similar to those in Western countries. The findings of the present study appear to justify the efforts to control total fat and SFA in the diet. Furthermore, the guidelines can expand on the specific food sources for complex carbohydrates and PUFA as they are desirable substitutes for SFA. Most of all, the issue of energy balance rather than the issue of low carbohydrates or of low fats should be considered, because it was also observed that unfavourable changes in chronic health conditions accompanied increased energy intake. The present study simply estimated the population-level consumption of different types of fat and the trends of the recent nine years. However, it is the first study to document population-level trends of nationally representative samples in Korea using the recently constructed comprehensive fatty acid database. This work can help shed light on public health concerns related to fat consumption and provide helpful information for setting adequate macronutrient distributions for the development of population-specific preventive strategies against dietrelated illness.

Author ORCID. (iD Jae Eun Shim, 0000-0001-8458-9112.

\section{Acknowledgements}

Financial support: This work was supported by the National Research Foundation of Korea (NRF) grant funded by the Korean Government (Ministry of Science and ICT; MSIT) (J.E.S., grant number 2016R1D1A1B03931820) (S.S., grant number 2017R1C1B5017637). The funder had no role in the design, analysis or writing of this article. Conflict of interest: None. Authorship: J.E.S. formulated the study question and performed the study design; S.S., J.E.S. and W.O.S. analysed the data; S.S., J.E.S. and W.O.S. drafted the manuscript and critically revised the manuscript; J.E.S. had primary responsibility for the final content. All authors contributed to the interpretation of the results and read and approved the final manuscript. Ethics of human subject participation: This study was conducted according to the guidelines laid out in the Declaration of Helsinki and all procedures involving human subjects were approved by the Korea Centers for Disease Control and Prevention Institutional Review Board. Written informed consent was obtained from all subjects.

\section{References}

1. Wong ND (2014) Epidemiological studies of CHD and the evolution of preventive cardiology. Nat Rev Cardiol 11, 276-289.

2. Keys A, Fidanza F, Scardi V et al. (1954) Studies on serum cholesterol and other characteristics of clinically healthy men in Naples. AMA Arch Intern Med 93, 328-336.

3. De Souza RJ, Mente A, Maroleanu A et al. (2015) Intake of saturated and trans unsaturated fatty acids and risk of all cause mortality, cardiovascular disease, and type 2 diabetes: systematic review and meta-analysis of observational studies. BMJ 351, h3978.

4. Harcombe Z, Baker JS, DiNicolantonio JJ et al. (2016) Evidence from randomised controlled trials does not support current dietary fat guidelines: a systematic review and metaanalysis. Open Heart 3, e000409. 
5. Harcombe Z, Baker JS \& Davies B (2017) Evidence from prospective cohort studies does not support current dietary fat guidelines: a systematic review and meta-analysis. $\mathrm{Br} \mathrm{J}$ Sports Med 51, 1743-1749.

6. Nettleton JA, Brouwer IA, Geleijnse JM et al. (2017) Saturated fat consumption and risk of coronary heart disease and ischemic stroke: a science update. Ann Nutr Metab $\mathbf{7 0}$, 26-33.

7. Chowdhury R, Warnakula S, Kunutsor S et al. (2014) Association of dietary, circulating, and supplement fatty acids with coronary risk: a systematic review and metaanalysis. Ann Intern Med 160, 398-406.

8. Skeaff CM \& Miller J (2009) Dietary fat and coronary heart disease: summary of evidence from prospective cohort and randomised controlled trials. Ann Nutr Metab 55, 173-201.

9. Li Y, Hruby A, Bernstein AM et al. (2015) Saturated fats compared with unsaturated fats and sources of carbohydrates in relation to risk of coronary heart disease: a prospective cohort study. J Am Coll Cardiol 66, 1538-1548.

10. Siri-Tarino PW, Sun Q, Hu FB et al. (2010) Meta-analysis of prospective cohort studies evaluating the association of saturated fat with cardiovascular disease. Am J Clin Nutr 91 , 535-546.

11. Mensink RP (2016) Effects of Saturated Fatty Acids on Serum Lipids and Lipoproteins: A Systematic Review and Regression Analysis. Geneva: WHO.

12. World Health Organization (2003) Diet, Nutrition and the Prevention of Chronic Diseases: Report of a Joint WHO/FAO Expert Consultation. WHO Technical Report Series no. 916. Geneva: WHO.

13. Popkin BM \& Du S (2003) Dynamics of the nutrition transition toward the animal foods sector in China and its implications: a worried perspective. J Nutr 133, 11 Suppl. 2, 3898S-3906S.

14. Popkin BM (2006) Global nutrition dynamics: the world is shifting rapidly toward a diet linked with noncommunicable diseases. Am J Clin Nutr 84, 289-298.

15. Yoon MO, Kim K, Hwang J-Y et al. (2014) Development of a fatty acids database using the Korea National Health and Nutrition Examination Survey data. J Nutr Health 47, 435-442.

16. Kweon S, Kim Y, Jang M-J et al. (2014) Data resource profile: the Korea National Health and Nutrition Examination Survey (KNHANES). Int J Epidemiol 43, 69-77.

17. Rural Development Administration, National Rural Resources Development Institute (2006) Food Composition Table, 7 th ed. Suwon, Republic of Korea: NRRDI.

18. Rural Development Administration, National Rural Resources Development Institute (2011) Food Composition Table, 8th ed. Suwon, Republic of Korea: NRRDI.

19. US Department of Agriculture, Agricultural Research Service, Nutrient Data Laboratory (2012) USDA National Nutrient Database for Standard Reference, Release 25. Beltsville, MD: USDA.

20. Ministry of Health and Welfare, Korea Center for Disease Control and Prevention (2016) Korea Health Statistics 2015: Korea National Health and Nutrition Examination Survey (KNHANES VI-3). Sejong, Republic of Korea: MoHW.

21. World Health Organization (2000) The Asia-Pacific Perspective: Redefining Obesity and Its Treatment. Sydney: Health Communications Australia.

22. Grundy SM, Cleeman JI, Daniels SR et al. (2005) Diagnosis and management of the metabolic syndrome: an American
Heart Association/National Heart, Lung, and Blood Institute scientific statement. Circulation 112, 2735-2752.

23. Popkin BM (1994) The nutrition transition in low-income countries: an emerging crisis. Nutr Rev 52, 285-298.

24. Popkin BM (2004) The nutrition transition: an overview of world patterns of change. Nutr Rev 62, 7 Pt 2, S140-S143.

25. Keys A (1953) Atherosclerosis: a problem in newer public health. Atherosclerosis 1, 19.

26. McGuire S (2011) US Department of Agriculture and US Department of Health and Human Services, Dietary Guidelines for Americans, 2010. Washington, DC: US Government Printing Office, January 2011. Adv Nutr 2, 293-294.

27. Center for Disease Control and Prevention (2004) Trends in intake of energy and macronutrients - United States, 1971-2000. MMWR Morb Mortal Wkly Rep 53, 80-82.

28. Wright JD \& Wang C-Y (2010) Trends in intake of energy and macronutrients in adults from 1999-2000 through 20072008. NCHS Data Brief issue 49, 1-8.

29. National Center for Health Statistics (2017) Health, United States, 2016: With Chartbook on Long-Term Trends in Health. Hyattsville, MD: NCHS.

30. Harcombe Z (2017) Dietary fat guidelines have no evidence base: where next for public health nutritional advice? $\mathrm{Br} \mathrm{J}$ Sports Med 51, 769-774.

31. Fryar CD, Wright JD, Eberhardt MS et al. (2012) Trends in nutrient intakes and chronic health conditions among Mexican-American adults, a 25-year profile: United States, 1982-2006. Natl Health Stat Report issue 50, 1-20.

32. Barclay AW, Petocz P, McMillan-Price J et al. (2008) Glycemic index, glycemic load, and chronic disease risk - a meta-analysis of observational studies. Am J Clin Nutr $\mathbf{8 7}$, $627-637$.

33. Liu S, Willett WC, Stampfer MJ et al. (2000) A prospective study of dietary glycemic load, carbohydrate intake, and risk of coronary heart disease in US women. Am J Clin Nutr $\mathbf{7 1}$, $1455-1461$.

34. Hooper L, Martin N, Abdelhamid A et al. (2015) Reduction in saturated fat intake for cardiovascular disease. Cochrane Database Syst Rev issue 6, CD011737.

35. Mozaffarian D, Micha R \& Wallace S (2010) Effects on coronary heart disease of increasing polyunsaturated fat in place of saturated fat: a systematic review and meta-analysis of randomized controlled trials. PLoS Med 7, e1000252.

36. Jakobsen MU, O'Reilly EJ, Heitmann BL et al. (2009) Major types of dietary fat and risk of coronary heart disease: a pooled analysis of 11 cohort studies. Am J Clin Nutr 89, $1425-1432$.

37. Hu FB, Stampfer MJ, Manson JE et al. (1997) Dietary fat intake and the risk of coronary heart disease in women. New Engl J Med 337, 1491-1499.

38. Grundy SM \& Vega GL (1988) Plasma cholesterol responsiveness to saturated fatty acids. Am J Clin Nutr $\mathbf{4 7}$, 822-824.

39. Katan MB, Zock PL \& Mensink RP (1995) Dietary oils, serum lipoproteins, and coronary heart disease. Am J Clin Nutr $\mathbf{6 1}$, 6 Suppl., 1368S-1373S.

40. Popkin BM (2001) Nutrition in transition: the changing global nutrition challenge. Asia Pac J Clin Nutr 10, Suppl, S13-S18.

41. Su C, Zhao J, Wu Y et al. (2017) Temporal trends in dietary macronutrient intakes among adults in rural China from 1991 to 2011: findings from the CHNS. Nutrients 9, 227. 